\title{
Low-Complexity Framework for Movement Classification Using Body-Worn Sensors
}

\author{
Dwaipayan Biswas, Koushik Maharatna, Member, IEEE, Goran Panic, Evangelos B. Mazomenos, \\ Josy Achner, Jasmin Klemke, Michael Jöbges, and Steffen Ortmann
}

\begin{abstract}
We present a low-complexity framework for classifying elementary arm movements (reach retrieve, lift cup to mouth, and rotate arm) using wrist-worn inertial sensors. We propose that this methodology could be used as a clinical tool to assess rehabilitation progress in neurodegenerative pathologies tracking occurrence of specific movements performed by patients with their paretic arm. Movements performed in a controlled training phase are processed to form unique clusters in a multidimensional feature space. Subsequent movements performed in an uncontrolled testing phase are associated with the proximal cluster using a minimum distance classifier (MDC). The framework involves performing the compute-intensive clustering on the training data set offline (MATLAB), whereas the computation of selected features on the testing data set and the minimum distance (Euclidean) from precomputed cluster centroids are done in hardware with an aim of low-power execution on sensor nodes. The architecture for feature extraction and MDC are realized using coordinate rotation digital computer-based design that classifies a movement in $(9 n+31)$ clock cycles, $n$ being number of data samples. The design synthesized in STMicroelectronics 130-nm technology consumed $5.3 \mathrm{nW}$ at $50 \mathrm{~Hz}$, besides being functionally verified up to $20 \mathrm{MHz}$, making it applicable for real-time highspeed operations. Our experimental results show that the system can recognize all three arm movements with average accuracies of $86 \%$ and $72 \%$ for four healthy subjects using accelerometer and gyroscope data, respectively, whereas for stroke survivors, the average accuracies were $67 \%$ and $60 \%$. The framework was further demonstrated as a field-programmable gate array-based real-time system, interfacing with a streaming sensor unit.
\end{abstract}

Index Terms-Activity recognition (AR), classification, clustering, coordinate rotation digital computer (CORDIC), field-programmable gate array (FPGA), low complexity.

\section{INTRODUCTION}

A CTIVITY recognition (AR) in nomadic settings has gained prominence in the research community for assessing human mobility through remote monitoring systems.

Manuscript received September 8, 2016; revised November 9, 2016; accepted December 14, 2016. This work was supported by the European Union within the Seventh Framework Programme under Grant \#288692, StrokeBack.

D. Biswas and K. Maharatna are with the Electronics and Computer Science Department, University of Southampton, Southampton SO17 1BJ, U.K. (e-mail: db9g10@ecs.soton.ac.uk; km3@ecs.soton.ac.uk).

G. Panic and S. Ortmann are with IHP, 15236 Frankfurt, Germany (e-mail: panic@ihp-microelectronics.com; ortmann@ihp-microelectronics.com).

E. B. Mazomenos is with the Centre for Medical Image Computing, University College London, London NW1 2PS, U.K. (e-mail: e.mazomenos@ ucl.ac.uk).

J. Achner, J. Klemke, and M. Jöbges are with Brandenburg Klinik, 16321 Berlin, Germany (e-mail: josy.achner@brandenburgklinik.de; ergo_n1@brandenburgklinik.de; joebges@brandenburgklinik.de).

Color versions of one or more of the figures in this paper are available online at http://ieeexplore.ieee.org.

Digital Object Identifier 10.1109/TVLSI.2016.2641046
Remote monitoring for long durations has been aided by the advancements ubiquitous and mobile computing facilities primarily using radio-frequency identification (RFID) [1], low-cost inertial sensors [2], and fusion of inertial sensor and vision-based approaches [3]. RFID and vision-based methods are primarily restricted to a defined region catering for indoor activities, requiring an unhindered surveillance [4]. Moreover, for systems requiring real-time information, using highcomplexity image processing algorithms can lead to slower analysis [3]. Hence, body-worn inertial sensors have gained prominence over other approaches [5], [6], particularly with the shift in research focus toward monitoring human activities performed in daily life, which is a more natural indicator of the subject's involvement compared with monitoring only during a prescribed exercise/training phase.

The fundamental requirement for a long-term continuous monitoring scenario using resource constrained wireless sensor network (WSN) nodes is a low-power operation to prolong the battery life. Typical remote monitoring systems employ computationally intensive data processing steps like feature extraction from the sensor data and pattern recognition (e.g., classification), which are carried out on offline computational facilities. This involves continuous data transmission incurring significant amount of energy expenditure at the radio front-end of the sensors. Hence, for applications involving continuous remote monitoring (e.g., motion/fall detection for the elderly population in daily life), a low-power strategy is of paramount importance, which can be achieved by performing low-complexity data processing in resource constrained environment of the sensor node itself [7].

In this paper, we focus on the application area of arm movement recognition aimed at stroke rehabilitation. In neurodegenerative pathologies (e.g., stroke or cerebral palsy), detecting and classifying particular arm movements (e.g., clinically prescribed exercises) performed in daily life can over time provide a measure of rehabilitation progress. A systematic exploration to recognize three fundamental movements of the upper limb associated with daily living activities using wrist-worn inertial sensors has already been reported in [8] employing a clustering and minimum distance classification-based approach. Sensor data collected from each subject in a constrained training phase (e.g., in the laboratory) are clustered to form three unique clusters representing each movement in a multidimensional feature space. A minimum distance classifier (MDC) computes the proximity of the test data collected in an unconstrained scenario (e.g., out of laboratory), to each of the clusters. 
Classification of the movements performed in the subsequent testing phase involves the essential steps of: 1) computing selected time-domain features from the sensor data and 2) the distance to the precomputed cluster centroids can be mapped to a low-complexity architecture to achieve real-time detection of arm movements, thereby providing an energy-efficient solution toward long-term operation of wearable sensors [7].

Hence, in this paper, we propose the design and implementation of a coordinate rotation digital computer (CORDIC)based low-complexity MDC for real-time arm movement recognition. The fundamental mathematical processes of the MDC have been formulated using the different transcendental functions realizable using CORDIC, and an optimized implementation strategy has been adapted, analyzing the shared computational stages. The algorithm proposed in [8] has been implemented in an offline-online resource sharing mechanism, where the time and memory intensive process of feature extraction, selection, and cluster formation using ten runs of tenfold cross validation (CV) on the training data was done in an offline mode (in MATLAB). The computation of the selected features (required for cluster formation) on the testing data and computation of the minimum distance (Euclidean) from the precomputed cluster centroids was done in hardware, targeting real-time implementation.

The design was synthesized using STMicroelectronics 130-nm technology with a supply voltage of $1.08 \mathrm{~V}$ and occupied $242 \mathrm{~K}$ NAND2 equivalent cell area and consumed $5.3 \mathrm{nW}$ at $50 \mathrm{~Hz}$, resulting in a low-complexity framework, applicable for real-time operations within a WSN node. The application area we consider is that of human AR where a sampling frequency of up to $50 \mathrm{~Hz}$ is deemed sufficient for capturing kinematic information [9], [10]. The design was further verified up to higher frequencies (namely, $20 \mathrm{MHz}$ ), and a total chip area of the layout was calculated as $2.21 \mathrm{~mm}^{2}$. Our experimental results to classify movements of four healthy subjects and stroke survivors involving an archetypal activity of daily living, 'making cup of tea,' show that the system can recognize all three arm movements with average accuracies of $86 \%$ and $72 \%$ for healthy subjects using accelerometer and gyroscope data, respectively, whereas for stroke survivors, the average accuracies were $67 \%$ and $60 \%$. The framework was further demonstrated as a real-time working system, interfacing a streaming inertial sensor unit, host PC, and DE4 field-programmable gate array (FPGA) board to facilitate serial port controls, recognizing a performed arm movement in approximately $0.6 \mathrm{~ms}$ at $780 \mathrm{KHz}$. The main contributions can be enlisted as the development of the following:

1) CORDIC-based low-complexity MDC architecture for online AR;

2) system demonstrator for real-time AR;

3) generic offline-online framework in conjunction with clustering, applicable in a wide range of AR applications.

The rest of this paper is structured as follows. An overview of the application setup is described in Section II, and the theoretical formulation of the MDC in terms of CORDIC rotation along with the architecture for the proposed framework is described in Section III. Section IV describes the

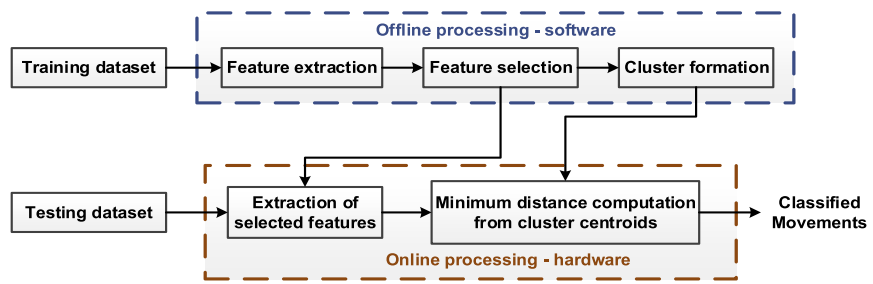

Fig. 1. Processing framework-offline/online processing of the training/ testing data set, respectively.

implementation and performance evaluation of the system. Finally, related literature and discussion are presented in Sections V and VI, respectively.

\section{Application Setup}

With an aim of continuous monitoring of activities performed in daily life by patients, the specific movements (or exercises) that need to be tracked as defined by clinicians need to be performed multiple times, following an exercise regime or a gaming session, in a controlled environment (clinic or home) [11], [12]. The sensor data collected during this phase can be analyzed through $\mathrm{CV}$ to determine the best cluster forming features and obtain the centroids of each cluster corresponding to each movement. This helps to perform a clinical profiling of the individual patient with respect to their movement quality. Movements performed in the uncontrolled nomadic environment (involving daily activities) can be associated with the proximal cluster centroid using the MDC to detect the occurrence of those particular movements. The merits of using the clustering based methodology over a plethora of other machine learning algorithms [10] for fine-grained arm movements have been presented in detail in [8], whereas issues such as sensor selection/placement and data fusion have been addressed in [13] for arm movement recognition.

Given the application framework, this methodology can be implemented for online detection of arm movements in a resource-constrained environment of body-worn sensor nodes. The offline processing of the training data, involving the key steps of cluster formation and feature selection, needs to be done only when requested by the clinician, depending on the rehabilitation progress of the patient over time. Furthermore, the test data can be classified in real time by computing the required features and the distance to the precomputed cluster centroids in near real time, providing an energy-efficient solution toward long-term operation of wearable sensors. The offline-online processing framework is illustrated in Fig. 1. Although here we have targeted arm movement as a case study, this framework can be suitably used for critical event monitoring such as fall detection or in sports medicine.

Experiments were conducted in two phases: training/ laboratory phase and a testing/out-of-laboratory phase on four healthy subjects at the University of Southampton and four stroke survivors at the Brandenburg Klinik. The healthy subjects were both right arm dominant, while the stroke population had either left or right arm impaired. For this investigation, three arm movements elementary in nature were considered: 1) Action A-reach and retrieve object; 2) Action B-lift 
TABLE I

Use Case ACtivity List- 'Making Cup of TeA'

\begin{tabular}{llc}
\hline \hline \multicolumn{1}{c}{ Activity } & Action \\
\hline 1. Fetch cup from desk & $\mathrm{A}$ \\
2. Place cup on kitchen surface & $\mathrm{A}$ \\
3. Fetch kettle & $\mathrm{A}$ \\
4. Pour out extra water from kettle & $\mathrm{C}$ \\
5. Put kettle onto charging point & $\mathrm{A}$ \\
6. Reach out for power switch on the wall & $\mathrm{A}$ \\
7. Drink glass of water while waiting for kettle to boil & $\mathrm{B}$ \\
8. Reach out to switch off kettle & $\mathrm{A}$ \\
9. Pour hot water from kettle in to cup & $\mathrm{C}$ \\
10. Fetch milk from shelf & $\mathrm{A}$ \\
11. Pour milk into cup & $\mathrm{C}$ \\
12. Put bottle of milk back on shelf & $\mathrm{A}$ \\
13. Fetch cup from kitchen surface & $\mathrm{A}$ \\
14. Have a sip and taste the drink & $\mathrm{B}$ \\
15. Have another sip while walking towards desk & $\mathrm{B}$ \\
16. Unlock the drawer & $\mathrm{C}$ \\
17. Retrieve biscuits from the drawer & $\mathrm{A}$ \\
18. Eat a biscuit & $\mathrm{B}$ \\
19. Lock the drawer & $\mathrm{C}$ \\
20. Have a drink & $\mathrm{B}$ \\
\hline \hline
\end{tabular}

cup to mouth; and 3) Action C-perform pouring/(un)locking action.

In the training phase, essential for the target cluster formation, each healthy participant performed 240, 120, and 120 trials each of Action A, Action B, and Action C, respectively. The stroke survivors performed 80 trials of $A$ and 40 trials each of $B$ and $C$. The collection of this training set helps to inherently capture the personalized (i.e., personcentric) movement patterns of the individuals through unique clusters augmenting accurate recognition [14]. The more number of trials pertaining to Action $A$ with respect to $B$ and $C$ is representative of the generalized nature of the reach and retrieve movement performed frequently in daily lives.

The testing phase employs an archetypal activity list (see Table I) emulating the process of 'making cup of tea,' commonly performed in daily life incurring repeated occurrences of the three investigated arm movements. The list comprises 20 individual activities having ten occurrences of Action $A$ and five each of Actions $B$ and $C$. The healthy subjects performed the activity list four times with a 10-min rest period between trials, whereas the stroke survivors performed two trials since they tend to tire quicker. The experiment was performed in an unconstrained manner ensuring wider range of variability in the data.

For this investigation, we use triaxial accelerometers (range $\pm 1.5 \mathrm{~g}$ ) and triaxial gyroscopes (range $\pm 500 \% \mathrm{~s}$ ), housed in a Shimmer wireless 9DoF kinematic sensor module [15]. The impaired arm for the stroke survivors and the dominant arm for the healthy subjects, proximal to the wrist, were chosen for the sensor placement with the dorsal side of the forearm in contact with the $X Y$ plane and the $Z$-axis pointing away from it. Magnetometers were not considered for this investigation due to the presence of ferromagnetic materials in the home environment [16]. Data were collected at $50 \mathrm{~Hz}$, transmitted along with a timestamp to a host computer using Bluetooth.

\section{Algorithm to Architecture Mapping}

The accuracy of any movement recognition technique is dependent on several factors such as nature/number of activities, sensor type/number/placement, data mining, and the classification methodology adopted [10]. Furthermore, there is a need for personalized evaluation, especially for tracking activities that are susceptible to individual and temporal variation. In this paper, although the focus is primarily on an optimized architecture design for the testing phase, a brief overview of the algorithm and associated data processing, especially in the training phase, is quintessential since it determines the generation of the cluster centroids used by the MDC. The $k$-means clustering algorithm mentioned in [8] uses ten time-domain features, extracted from each of the three axes of the accelerometer or the gyroscope sensors. The features are: 1) standard deviation; 2) root mean square; 3) information entropy; 4) jerk metric; 5) peak number; 6) maximum peak amplitude; 7) absolute difference; 8) index of dispersion; 9) kurtosis; and 10) skewness.

The fundamental concept of clustering is to form groups of similar objects as a means of distinguishing them from each other, and it is well perceived that cluster analysis is primarily used for unsupervised learning where the class labels for the training data are unknown. However, $k$-means clustering can also be used for supervised learning as in our proposed methodology [8] where we are aware of the labels for the training data pertaining to the three movements, helping to have a definite estimate on the underlying cluster structure (three clusters), facilitating faster convergence during cluster formation. We use the regularized Mahalonobis distance considering the covariance of the data, where a parameter $\lambda$ ( 0 or 1 ) is used to control the choice of distance measure (squared Mahalonobis or Euclidean). The clustering is performed on the feature vectors computed from the training data (accelerometer and gyroscope). It is performed in conjunction with a sequential forward selection algorithm, selecting a combination of 2-30 ranked features (ten features computed on each triaxial axes' data) in each step, and ten runs of tenfold CV (nine segments of training and one of testing - only considering training data) are carried out with each feature combination. Cluster centroids are selected based on an experimentally determined threshold (25\%) of the expected number of data points for each of the three clusters formed (healthy subjects: Action $A-240 \pm 60$, Action B/C $-120 \pm 30$; patients: Action $A-80 \pm 20$, Action $B / C-40 \pm 10$ ). Therefore, offline processing provides a detailed list of feature combinations that resulted in a successful cluster formation and the highest corresponding accuracies (averaged over ten runs) for each subject and each sensor type.

An important aspect is the choice of features since human AR studies typically incur the extraction of time and/or frequency domain features, as well as heuristic features from data which exhibit discriminative patterns for each movement. Commonly used frequency-domain features as a result of signal transformation-using Fourier and wavelet-are well 
equipped in capturing dynamic movements like walking, running, and so on (high-frequency components), while the orientation/postural information can be obtained from the lowfrequency components. In this investigation, we use timedomain features since: 1) we consider fine-grain upper limb movement compared with detecting gross/dynamic activities and static postures like standing, running, sitting, and cycling and 2) time-domain feature extraction incurs low complexity when mapped onto equivalent architecture.

A detailed study on sensor selection/placement and data fusion for the targeted arm movements has been reported in [13]. In particular, it has been shown that higher recognition sensitivities are achieved using: 1) data from the wrist sensor module compared with the elbow since the former is more responsive and produces significant discriminatory patterns for the arm movements being investigated and 2) similar timedomain features extracted on individual sensor axes' data compared with considering he modulus of the triaxial data and fusion of specific accelerometer-gyroscope signal combinations. Three unique sensor combinations for the wrist module (multiplying accelerometer-gyroscope signals) were created based on a priori consideration of the expected trajectory of the subject's arm with respect to the sensor position and orientation of the sensor axes for the investigated movements. The use of all the individual sensor signals, rather than a processed signal (i.e., moduli or fused), provides the classifier a wider pool of features to select, and hence the recognition rate for the movements is reflected in the higher sensitivity achieved [13].

According to the application framework (see Section II), the online processing stage aimed at real-time arm movement detection comprises the key steps: 1) feature extraction from the test data set and 2) associating the test data with the precomputed cluster centroids using an MDC. In this section, we present the architecture and implementation of the MDC in conjunction with the cluster centroids for detecting the three investigated arm movements. A detailed architecture and implementation of several of these features have been presented in [17], except for the jerk metric that is an important feature, quantifying the tremor inherent in the movement, especially among the stroke population. Given the low-complexity when using CORDIC for formulating the features as demonstrated in [17] compared with other implementations, in this paper, we use it to formulate: 1) the jerk metric and 2) the MDC for classifying the test data in the respective feature space. We present a brief overview of CORDIC fundamentals, used for the algorithmic-to-architecture formulation.

CORDIC is an iterative algorithm that uses 2-D vector rotation for computing different transcendental functions employing the iterative equations

$$
\begin{aligned}
& x_{j+1}=x_{j}-\mu \sigma_{j} \cdot 2^{-j} \\
& y_{j+1}=y_{j}-\sigma_{j} \cdot 2^{-j} \cdot x_{j} \\
& z_{j+1}=x_{j}-\sigma_{j} \cdot \alpha_{j}
\end{aligned}
$$

where $\left[x_{j}, y_{j}\right]^{T}, z_{j}$, and $\sigma_{j} \epsilon\{1,-1\}$ are the intermediate result vector, the residual angle, and the direction of vector rotation at the $j$ th iteration stage, respectively, $\mu \epsilon\{1,0\}$ being the coordinate of rotation-circular and linear, respectively.
TABLE II

Generalized CORDIC Algorithm In Two CoORdinate Systems

\begin{tabular}{ccc}
\hline \hline$\mu$ & ROTATION MODE $\left(Z_{0} \rightarrow 0\right)$ & VECTORING $\left(Y_{0} \rightarrow 0\right)$ \\
\hline & $x_{n}=K\left(x_{0} \cos z_{0}-y_{0} \sin z_{0}\right)$ & $x_{n}=K \sqrt{{x_{0}{ }^{2}+y_{0}^{2}}^{2}}$ \\
1 & $y_{n}=K\left(y_{0} \cos z_{0}+y_{0} \sin z_{0}\right)$ & $y_{n}=0$ \\
$z_{n}=0$ & $z_{n}=z_{0}+\tan ^{-1}\left(y_{0} / x_{0}\right)$ \\
& $x_{n}=x_{0}$ & $x_{n}=x_{0}$ \\
0 & $y_{n}=y_{0}+x_{0} z_{0}$ & $y_{n}=0$ \\
& $z_{n}=0$ & $z_{n}=z_{0}+\left(y_{0} / x_{0}\right)$ \\
\hline \hline
\end{tabular}

In each coordinate system, CORDIC, in general, can be operated in two modes-vectoring and rotation [18]. For an input vector $\left[\begin{array}{ll}x_{0} & y_{0}\end{array}\right]^{T}$, in the vectoring mode $\left(y_{0} \rightarrow 0\right)$, the magnitude of the vector and angle between the initial vector and the principal coordinate axis are computed, whereas in the rotation mode $\left(z_{0} \rightarrow 0\right)$, for a given angle of rotation, the final vector is computed. These can be used for computing a series of transcendental functions as shown in Table II [18]. The transcendental functions generated by the vectoring CORDIC operation can be used for feature computation and the MDC. We use $\mathrm{Vec}_{c}$ and $\mathrm{Vec}_{l}$ as operators representing vectoring CORDIC operation in circular and linear coordinate systems, respectively. The input data set is represented by $d_{s i}$, where $i \epsilon\{0,1,2 \ldots n-1\}$ and $d_{i}$ is the output of vectoring CORDIC operation on $d_{s(i-1)}$ data sample. The features and the MDC have been formulated in terms of CORDIC operation, in line with this convention.

\section{A. Feature-Jerk Metric}

The jerk metric (jm) characterizes the average rate of change of acceleration in a movement. It is calculated as the rms value of the derivative of the acceleration (jerk) normalized by the maximum value of the integral (velocity) [19] as shown in

$$
\text { jerk metric }=\frac{\operatorname{rms}\left[\frac{d\left(d_{s i}\right)}{d t}\right]}{\max \left[\int\left(d_{s i}\right) d t\right]} .
$$

It is important to note here that although the calculation of jerk is physically related to the acceleration data, the same computing logic is also applied to the rotation data from the gyroscope, since the computed metric serves its purpose as a discriminating feature for characterizing the movements. Since the data samples are equally spaced due to the constant sampling frequency, the first derivative is computed as the difference of the consecutive data samples using a subtractor. The integral of the data is computed using trapezoidal integration that involves the addition of the consecutive data samples and a divide by two (implemented as right shift). From (2), it can be deduced that the rms of the first derivative of the data samples $\left(\dot{d}_{s i}\right)$ can be computed using the operator $\mathrm{Vec}_{c}$, which is shown in (3). The samples $\dot{d}_{s i}$ are used as the $y$ input to the CORDIC and the $x$-component of the output is fed back to the $x$-component of the CORDIC input

$$
\mathrm{rms}=\frac{1}{\sqrt{n}}\left(\prod_{i=0}^{n-1} \operatorname{Vec}_{c}\left[\begin{array}{ll}
d_{i} & \dot{d}_{s i}
\end{array}\right]^{T}\right)_{x}
$$


Therefore, with new data samples $d_{s i}$ arriving at each clock cycle, the $x$-component of the CORDIC output is computed as

$$
d_{i}=K \sqrt{d_{s 0}^{2}+d_{s 1}^{2}+\cdots+d_{s(i-1)}^{2}} .
$$

The $x$-component of the output generated after every complete CORDIC operation is scaled with a scale factor $K$. This is an essential step as feeding this result without scaling into the $x$-component of the CORDIC input results in an accumulation of the scale factor corresponding to each $d_{s i}$, thereby affecting the formulation in (4). Hence, the scale factor compensation is invoked after every complete CORDIC operation (comprising $N$ stages) with a set of input data, feeding the compensated output to the $x$-input of the CORDIC in the next iteration. Following $n$ operations with the scale factor compensation, the $x$ output of the CORDIC yielding the final result is multiplied with $1 / \sqrt{ } n$ for obtaining the rms. The value $1 / \sqrt{ } n$ is precomputed ( $n$ being a fixed number) and is multiplied with the final CORDIC output with the help of a reduced complexity multiplier-less shift-and-add technique or fixednumber multiplier.

The jerk metric is finally computed using the CORDIC operator $\mathrm{Vec}_{l}$ as shown in (5). Referring to Table I, $\max \left(\int d_{s i}\right)$ and $\operatorname{rms}\left(\dot{d}_{s i}\right)$ are set as the $x_{0}$ and $y_{0}$ inputs to the CORDIC, operating in vectoring mode in the linear coordinate system

$$
\text { jerk metric }=\left(\operatorname{Vec}_{l}\left[\max \left(\int d_{s i}\right) \operatorname{rms}\left(\dot{d}_{s i}\right)\right]^{T}\right)_{z}
$$

The implementation includes one subtractor and CORDIC $\left(\operatorname{Vec}_{c}\right)$ for computing $\operatorname{rms}\left(\dot{d}_{s i}\right)$ and one adder for computing $\left(\int d_{s i}\right)$ by trapezoidal integration. Finally, CORDIC $\left(\mathrm{Vec}_{l}\right)$ is reused for computing the value of the feature. The jerk metric is dependent on the rms of the derivative and maximum of the integral taking $(n+1)$ cycles. Considering $n$ as 256 data samples, representative of a movement for approximately $5 \mathrm{~s}(50 \mathrm{~Hz})$, facilitates a multiplier-less shift-and-add operation.

\section{B. Minimum Distance Classifier}

The MDC methodology has been illustrated through a mathematical approach having three clusters $(A, B$, and $C$ formed using $k$-means on the training data set for the three movements) and a test vector $(T)$ to be associated in a 2-D feature space $\left(f_{1}\right.$ and $\left.f_{2}\right)$ in Fig. 2. The distance of $T$ from each of the three centroids are denoted by $d_{A}, d_{B}$, and $d_{C}$, which are compared to estimate its proximity to the clusters.

According to Fig. 2, the 2-D coordinates are cluster centroid $A-\left(f_{A 1}, f_{A 2}\right)$ and test vector $T-\left(f_{T 1}, f_{T 2}\right)$. This feature space $\left(f_{1}, f_{2}\right)$ can be extended to incorporate all 30 features. The Euclidean distance of the test feature vectors from the centroid can be computed as in (6), which can be further reframed (7), having functional similarity to rms computation and can be realized using CORDIC operator $\operatorname{Vec}_{c}(8)$, where the data samples $d_{A s i}$, are the computed differences between

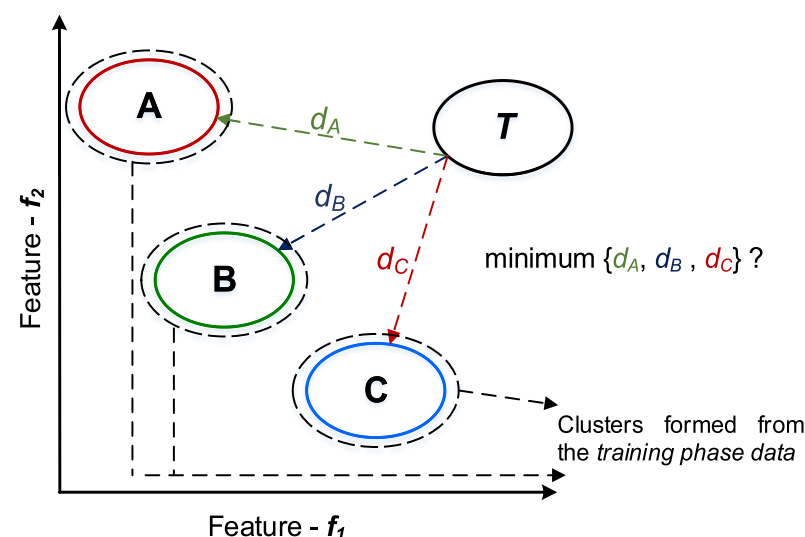

Fig. 2. Illustration of the minimum distance classification methodology.

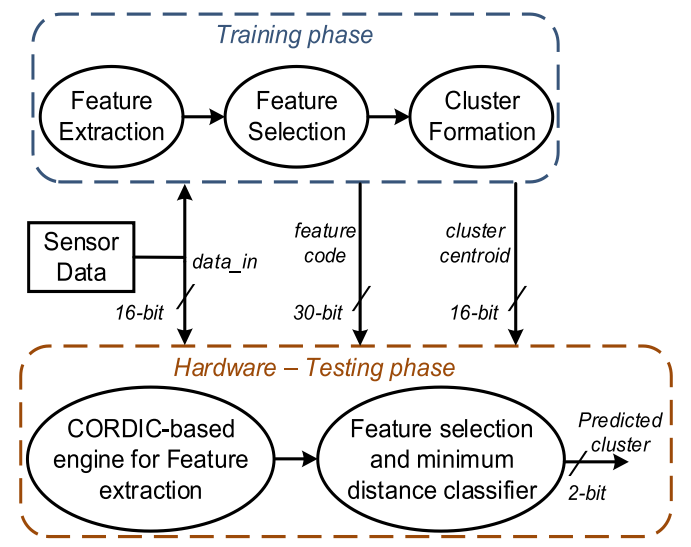

Fig. 3. Architecture for offline-online framework for MDC.

the feature vectors of the test data set and the cluster centroids $d_{A}=\sqrt{\left(f_{T 0} \sim f_{A 0}\right)^{2}+\left(f_{T 1} \sim f_{A 1}\right)^{2}+\cdots+\left(f_{T 29} \sim f_{A 29}\right)^{2}}$

$d_{A}=\sqrt{\left(\sum_{i=0}^{29} d_{A s i}^{2}\right)}$

$d_{A}=\left(\prod_{i=0}^{n-1} \operatorname{Vec}_{c}\left[d_{A i} d_{A s i}\right]^{T}\right)_{x}$.

Similar to rms computation (3), the samples $d_{A s i}$ are fed to the $y$ input of CORDIC while the final result (scaled with $K$ ) at the $x$ output of CORDIC is obtained after $n$ number of operations, where $n$ is dependent on the number of features selected $(1>n \leq 30)$. Similarly, the distances $d_{B}$ and $d_{C}$ can be computed using $\mathrm{Vec}_{c}$. The offline-online processing approach (see Section II) has been illustrated in Fig. 3, representing the input-output signals that have been further described in Table III.

The sequence of features (ten features) has been illustrated in Fig. 4, which are extracted from each triaxial data segment $(x, y$, and $z)$ of each sensor type, thereby having a total of 30 features [8]. The features selected (out of a total of 30) during the cluster formation are represented using a feature code. An example 30-bit code: 000100000000000001001000000000 
TABLE III

LIST OF INPUT-OUTPUT SIGNALS

\begin{tabular}{|c|c|}
\hline Signals & Description \\
\hline data_in & $\begin{array}{l}\text { 16-bit } \mathrm{i} / \mathrm{p} \text { for tri-axial sensor data corresponding to a } \\
\text { movement performed in the testing phase (Acc_x, Acc_y, } \\
\text { Acc_z or Gyro_x, Gyro_y, Gyro_z) }\end{array}$ \\
\hline feature-code & $\begin{array}{l}\text { 30-bit } \mathrm{i} / \mathrm{p} \text { denoting the selected features out of total } 30 \\
\text { features during cluster formation on the training dataset } \\
\text { (having ' } 1 \text { ' for a selected feature else ' } 0 \text { '); cf. Fig. } 4 \text {. }\end{array}$ \\
\hline $\begin{array}{l}\text { cluster- } \\
\text { centroid }\end{array}$ & $\begin{array}{l}16 \text {-bit } \mathrm{i} / \mathrm{p} \text { each for } 3 \text { cluster centroids formed from the } \\
\text { features selected from the training phase data. }\end{array}$ \\
\hline $\begin{array}{l}\text { predicted- } \\
\text { cluster }\end{array}$ & $\begin{array}{l}\text { 2-bit } \mathrm{o} / \mathrm{p} \text { for the predicted cluster computed as the minimum } \\
\text { distance of the test dataset from the cluster centroids }\end{array}$ \\
\hline
\end{tabular}

\begin{tabular}{|c|c|c|c|c|c|c|c|c|c|}
\hline rms & abs. diff & $\sigma$ & D & kurtosis & skew & inf. entropy & jerk & peaks & max_mag \\
\hline 0 & 1 & 2 & 3 & 4 & 5 & 6 & 7 & 8 & 9 \\
\hline
\end{tabular}

Fig. 4. Sequence of features extracted from each triaxial data segment to form a 30-bit feature code.

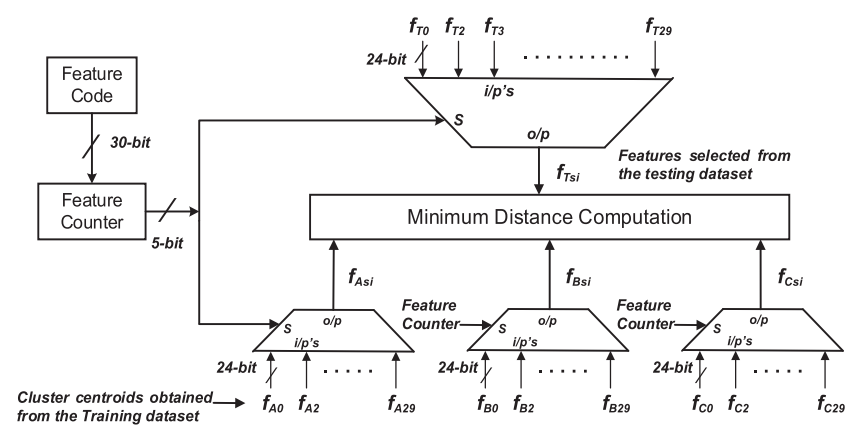

Fig. 5. Overview of the MDC architecture.

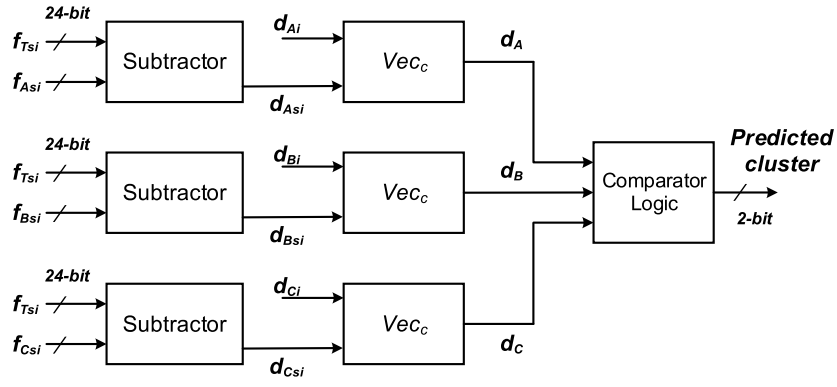

Fig. 6. Architecture for the minimum distance computation module.

represents the features $(3,17,20)$, namely, $D \_x$ (dispersion computed on the $x$-axis data), jerk_y (jerk metric on the $y$-axis), and $r m s_{-} z$ (rms on the $z$-axis) were selected during cluster formation.

The architecture for the MDC associating the test data set with precomputed cluster centroids is shown in Figs. 5 and 6. The feature code helps to select the required features. The cluster centroid for that corresponding feature is selected through a sample counter (5-bit feature counter), which counts through the 30-bit feature code.

The features selected from the testing set $\left(f_{T s i}\right)$ and the corresponding cluster centroids $\left(f_{A s i}, f_{B s i}\right.$, and $\left.f_{C s i}\right)$ are passed onto the minimum distance computation module, shown in Fig. 6, using subtractors to compute the difference between the corresponding features and cluster centroids that are used to compute the distance $\left(d_{A}, d_{B}\right.$, and $\left.d_{C}\right)$ using operator $\operatorname{Vec}_{c}$ (7) to produce the respective distances of the test set from each centroid. A comparator is used to determine the proximal cluster, denoted by 2-bit output ('00'-A, ' 01 ' $-B$, and ' 10 ' $-C$ ).

Here, we have used three CORDIC operations in parallel for distance computation from each centroid (see Fig. 5) that could be achieved by reusing one CORDIC module for a sequential computation but at the expense of an increased computation time. A high-speed design has been preferred in view of real-time detection. Using multiple CORDIC modules has its effects on the chip area and power, and hence a tradeoff with the computation time is necessary for an optimal design. In the worst case scenario, if all 30 features are selected, the distance computation from each of the three centroids would involve 30 CORDIC operations. Reusing a single CORDIC incurs additional processing time, along with the overheads of a control logic. The feature extraction engine consumes approximately $1 \mathrm{nW}$ of power [17] given the low-frequency operations $(50 \mathrm{~Hz})$, and therefore computation time has been given priority in this design.

The computation of the features and the MDC incurs a recursive formulation that leads to a computing loop that cannot be achieved with a pipelined CORDIC architecture, whereas using an iterative CORDIC implementation would have its effect on the throughput. Hence, a unit latency design coalescing all iterations in a single computing stage (one clock cycle) is adopted here. We present an estimate of the hardware complexity in terms of the total full adder (FA) count, which provides an objective reflection of the underlying architecture. The MDC requires three subtractors and three CORDIC modules $\left(\mathrm{Vec}_{c}\right)$. A $b$-bit ripple carry adder/subtracter requires $b$ FAs, and therefore we can consider $3 b$ FAs for the subtractors. The hardware resource for one iteration of an $N$-stage CORDIC rotation (considering a generalized word length $b$ ) can be computed as $2 \mathrm{Nb}$ FAs. This can be reused for multiple iterations (e.g., rms computation). Although the $M D C$ requires three CORDIC modules in parallel, two modules used for feature extraction (one module used for std, rms, entropy, dispersion, kurtosis, and skewness as reported in [17] and one module for jerk metric that is independent of the rest of the features) can be reused for MDC. Hence, in total, we require $(2 \mathrm{Nb}+3 b)$ FAs for the MDC implementation. For MDC implementation having 16-stage $(N)$ and 24-bit $(b)$ datapath, we require $840 \mathrm{FAs}$. It is important to note here that for the complexity analysis, we did not consider the comparator, the counter logic, and the multiplexers.

The complexity of an alternate architecture (without CORDIC) for MDC implementation can be estimated considering a squaring unit, nonrestoring iterative cellular square rooter (SQRT) [20], an accumulator (replacing one CORDIC module for the root-mean-square operation), and three subtractors. Hence, in total, it requires three squarers, three SQRTs, three accumulators, and three subtractors. For the sake of convenience, two squaring units can be considered as one 
multiplier and an accumulator block can be considered as an FA (registers associated with the accumulator are not considered, accounting only for arithmetic operations).

A conventional array multiplier (CAM) requires $b(b-2)$ FAs, $b$ half adders (HAs), and $b^{2}$ AND gates. Considering two HAs as one FA and four AND gates as one FA (due to area and transistor count), the total gate count of a CAM can be deduced as $\left(1.25 b^{2}-1.5 b\right)$ FAs. Hence, for three squaring units (1.5 CAM), $A_{m u l t}=1.5\left(1.25 b^{2}-1.5 b\right)$ FAs, where $\left(A_{*}\right)$ represents the total number of FAs in each circuit. A $b$-bit SQRT requires $0.125 \times b(b+6)$ FAs and similar number of XOR gates. Therefore, the total FA count for three SQRTs (considering two XOR gates as one FA) is $A_{S Q R T}=$ $\left(0.1875 b^{2}+1.125 b\right)$ FAs. Finally, $A_{a d d} / s u b=6 b$ FAs (three subtractors + three accumulators) are required. Therefore, the total gate count for the MDC computation using an alternate architecture in terms of FA count is $\left(A_{\text {mult }}+A_{S Q R T}+A_{\text {add } / \text { sub }}\right)=\left(2.0625 b^{2}+4.875 b\right)$ FAs. Hence, for a 24-bit datapath, we require 1305 Fas, which is more than the CORDIC-based implementation.

It is worthwhile to recollect here that the CORDIC-based feature extraction [17] engine requires $4110 \mathrm{FAs}$ (for 16-stage CORDIC and 24-bit datapath), whereas the non-CORDIC feature extractor requires 6828 FAs. Hence, even if the circuit elements from the non-CORDIC feature extractor are reused for its equivalent MDC implementation, a unified CORDICbased feature extraction engine and its equivalent MDC implementation will incur low complexity and result in an optimized design.

Another important factor is the effect of normalization. The clusters are formed in a multidimensional feature space where the cluster analysis takes place on the features extracted from the training data. These features are linearly normalized with respect to their minimum and maximum values. Therefore, the cluster centroids are represented by the normalized values (i.e., in the numeric range of $0-1$ ) of the selected features. However, during the testing phase, the relevant features are extracted from the corresponding sensor data using the feature extraction engine and used by the MDC lie in different numeric ranges compared with the respective centroids. Therefore, prior to computing the Euclidean distance, the centroids are unnormalized and used as inputs to the RTL module.

\section{IMPLEMENTATION AND EVALUATION}

\section{A. Verification}

The architecture for feature extraction and MDC was coded using Verilog as HDL with a target ASIC implementation. It is important to note here that although the input data is 16-bit wide, the datapath width in the CORDIC-based feature extraction engine and the MDC module is 24 bits. In order to achieve the desired 16-bit accuracy, a 22-bit word length should be selected [21], according to the formulation $\left(N+\log _{2} N+2\right)$ and at least 16 iterations. Therefore, to obtain a high accuracy, a 24-bit CORDIC was used for this implementation. The design was functionally verified using data of four healthy subjects and four stroke survivors. For each healthy subject, there were 80 test vectors (four trials of 'making cup of tea', having 20 movements in each trial).
TABLE IV

RECOGNition SEnSitivities For ARM MOVEMENTS of HeAlthy SubJects

\begin{tabular}{|c|c|c|c|c|c|}
\hline \multirow{2}{*}{ Subject } & \multirow{2}{*}{ Features } & \multicolumn{3}{|c|}{ Sensitivities (\%) } & \multirow{2}{*}{$\begin{array}{c}\text { Overall accuracy } \\
(\%)\end{array}$} \\
\hline & & $\boldsymbol{A}$ & $\boldsymbol{B}$ & $C$ & \\
\hline \multicolumn{6}{|c|}{ RTL Evaluation } \\
\hline \multicolumn{6}{|c|}{ Accelerometer } \\
\hline Subject1 & 11 & 100 & 100 & 75 & 94 \\
\hline Subject2 & 2 & 85 & 55 & 85 & 78 \\
\hline Subject3 & 7 & 90 & 90 & 90 & 90 \\
\hline Subject4 & 23 & 85 & 90 & 70 & 83 \\
\hline \multicolumn{6}{|c|}{ Gyroscope } \\
\hline Subject1 & 10 & 50 & 80 & 100 & 70 \\
\hline Subject2 & 27 & 70 & 80 & 70 & 73 \\
\hline Subject3 & 18 & 80 & 85 & 90 & 84 \\
\hline Subject4 & 20 & 40 & 90 & 75 & 61 \\
\hline \multicolumn{6}{|c|}{ Software Evaluation [8] } \\
\hline \multicolumn{6}{|c|}{ Accelerometer } \\
\hline Subject1 & 11 & 100 & 100 & 100 & 100 \\
\hline Subject2 & 2 & 80 & 5 & 80 & 61 \\
\hline Subject3 & 7 & 95 & 100 & 90 & 95 \\
\hline Subject4 & 23 & 95 & 100 & 85 & 94 \\
\hline \multicolumn{6}{|c|}{ Gyroscope } \\
\hline Subject1 & 10 & 93 & 90 & 100 & 94 \\
\hline Subject2 & 27 & 100 & 80 & 60 & 85 \\
\hline Subject3 & 18 & 90 & 90 & 100 & 93 \\
\hline Subject4 & 20 & 30 & 95 & 85 & 60 \\
\hline
\end{tabular}

Similarly, for each stroke survivor, there were 40 test vectors (two trials of 'making cup of tea'). The results using the accelerometer and the gyroscope data are shown in Tables IV and V for healthy subjects and stroke survivors. The software evaluation results (MATLAB) [8] are presented for comparison.

Stroke survivors 1 and 4 represent two extreme conditions (late and early stage of recovery after stroke) as evaluated by respective clinicians. Overall, the results of the RTL simulation are on the lower side when compared with the software evaluation. The average difference in accuracy between RTL and software simulation is $1.25 \%$ and $11 \%$ using accelerometer and gyroscope data, respectively, for healthy subjects. Similarly, for stroke survivors, the average difference in accuracy is $3.75 \%$ and $5.5 \%$ for the two sensor types, respectively. The difference in the results (decrease in individual movement sensitivities and the overall accuracy) of the RTL implementation and software can be attributed to the following factors.

1) Accumulation of truncation error, a common phenomenon in fixed-point arithmetic operations and occurs due to the implemented logic. Moreover, the software implementation (MATLAB) presents the results in a 64-bit operating system, whereas the CORDIC-based RTL module has a datapath width of 24 bits. Since, in this implementation, to achieve 16-bit accuracy, 16 iterations are used, and hence this recursive CORDIC operation results in error accumulation to a higher degree. Hence, for the MDC, where a data point is being classified based on a distance value, this accumulated error could result in misclassification. On the 
TABLE V

RECOGNITION SENSITIVITIES FOR ARM MOVEMENTS OF STROKE SURVIVORS

\begin{tabular}{|c|c|c|c|c|c|}
\hline \multirow{2}{*}{ Subject } & \multirow{2}{*}{ Features } & \multicolumn{3}{|c|}{ Sensitivities (\%) } & \multirow{2}{*}{$\begin{array}{c}\text { Overall accuracy } \\
(\%)\end{array}$} \\
\hline & & $A$ & $B$ & $C$ & \\
\hline \multicolumn{6}{|c|}{ RTL Evaluation } \\
\hline \multicolumn{6}{|c|}{ Accelerometer } \\
\hline Subject1 & 19 & 70 & 80 & 100 & 80 \\
\hline Subject2 & 19 & 85 & 20 & 100 & 73 \\
\hline Subject3 & 21 & 80 & 90 & 30 & 70 \\
\hline Subject4 & 8 & 20 & 80 & 50 & 43 \\
\hline \multicolumn{6}{|c|}{ Gyroscope } \\
\hline Subject1 & 8 & 80 & 60 & 80 & 75 \\
\hline Subject2 & 10 & 60 & 90 & 50 & 65 \\
\hline Subject3 & 24 & 80 & 20 & 70 & 63 \\
\hline Subject4 & 30 & 50 & 50 & 0 & 38 \\
\hline \multicolumn{6}{|c|}{ Software Evaluation [8] } \\
\hline \multicolumn{6}{|c|}{ Accelerometer } \\
\hline Subject1 & 19 & 80 & 90 & 100 & 88 \\
\hline Subject2 & 19 & 90 & 20 & 100 & 75 \\
\hline Subject3 & 21 & 95 & 100 & 20 & 78 \\
\hline Subject4 & 8 & 10 & 80 & 60 & 40 \\
\hline \multicolumn{6}{|c|}{ Gyroscope } \\
\hline Subject1 & 8 & 90 & 50 & 100 & 83 \\
\hline Subject2 & 10 & 60 & 100 & 60 & 70 \\
\hline Subject3 & 24 & 85 & 30 & 80 & 70 \\
\hline Subject4 & 30 & 60 & 40 & 0 & 40 \\
\hline
\end{tabular}

other hand, healthy subject2, requiring the computation of minimum number of features, namely, two, is an exception as the overall accuracy achieved is higher with RTL. The accumulated error in this case (for computing the two required features-standard deviation and root mean square computed on the $y$-axis data [8]) could have created a bias for the distance computation of the test data with respect to the centroids, thereby affecting the classification results yielding a higher accuracy. This effect is also observed to a less extent for the following subject/action/sensor combinations: healthy-2/C/gyroscope; 4/A/gyroscope and stroke1/B/gyroscope; 3/C/accelerometer; 4/A/accelerometer; and $4 / B /$ gyroscope. As further illustration, variation of recognition accuracies with respect to features for healthy subject 2 with accelerometer data (see Fig. 7) shows that using more features (beyond 2) does not result in successful cluster formations (blank spaces) or improved accuracy.

2) The difference of accuracy is further evident, especially while computing a higher number of features. There are more number of test data sets for healthy subjects compared with the stroke survivors and the high number of features computed (e.g., 30 for stroke survivor4 further contributes toward the mathematical error). It is evident from the feature computation engine [17] that the average error may become significant for the features particularly involving higher order terms (e.g., kurtosis and skewness) even when the accuracy of

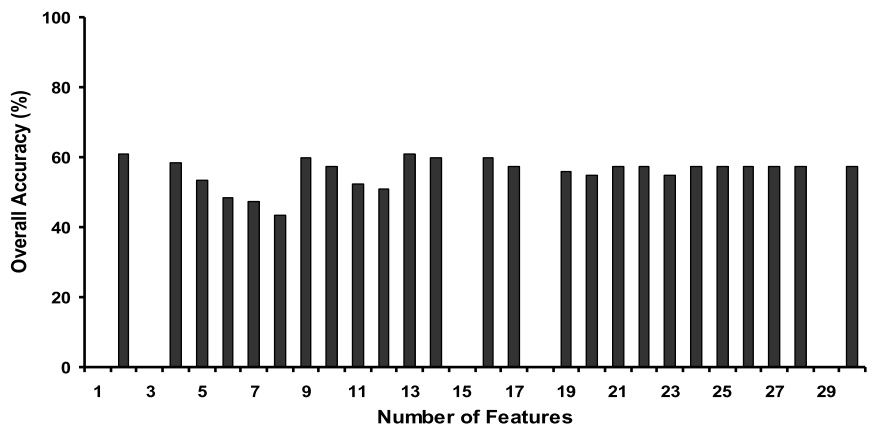

Fig. 7. Variation in accuracy with number of features for healthy Subject2 with accelerometer data during software evaluation [8].

the CORDIC itself is set high. Hence, to achieve higher accuracy, adjusting the datapath width for the MAC unit may be necessary depending on error tolerance of the application. A ranked list of the associated features for each subject chosen during cluster formation as a result of $\mathrm{CV}$ is presented in [8]. The number of features selected for each subject represents the optimal number of top ranked features that resulted in successful cluster formation and highest $\mathrm{CV}$ accuracies on the training data set.

3) In this implementation, a signal length of 256 data samples has been considered, which can be represented on a dyadic scale, and therefore any multiplication or division operation can be implemented through a shift. Hence, for testing with data already collected during the experimental protocol, an interpolation/extrapolation module in MATLAB was implemented to preprocess the test data to restrict the sample size to 256 as opposed to the software implementation.

4) Finally, in this design, we have not filtered the raw sensor data (preprocessing step [8]), to keep the computations at a minimal level. Here, our focus was mainly on the implementation of the MDC, and hence a filter block could be added to improve performance.

The achieved results, for both the healthy subjects (average accuracy of $86 \%$ and $72 \%$ with accelerometer and gyroscope, respectively) and the stroke survivors (average accuracy of $67 \%$ and $60 \%$ with accelerometer and gyroscope, respectively), can be considered favorable because the methodology was tested to detect activities performed in out-of-laboratory seminaturalistic scenario, having a significant degree of variability. The accuracy rates reported for the stroke survivors are acceptable, according to clinicians, since it provides a gross measure of impaired arm use. It is important to mention here that a misclassification of a performed movement may not have significant clinical impact because in this application (as opposed to other clinically critical remote monitoring applications, e.g., cardiovascular disease), the final decision on the rehabilitation measure and the corresponding prescription lies with the jurisdiction of the respective clinicians. This methodology could help to augment the clinical findings and provide a quantitative measure on the rehabilitation progress of patients over time outside the clinical environment. 
In view of the RTL simulation results, the conclusions drawn in [8] are still evident: 1) variability in data patterns due to poor repeatability and 2) considering more than one sensor type for specific cases can improve overall detection accuracy. This can be observed particularly for healthy subject 2 , where although the overall accuracy with accelerometer is $78 \%$, the sensitivity for Action $B$ is low (55\%), which is significantly improved when considering the gyroscope $(80 \%)$. Similar trends are observed for healthy subject 4 with Action A using gyroscope $(40 \%)$, which can be detected successfully when considering the accelerometer data (85\%). Considering more than one sensor type could be beneficial for stroke survivors as can be seen for the following subject/action combinations$2 / C$ (gyroscope-50\% and accelerometer-100\%) and $3 / C$ (accelerometer-30\% and gyroscope-70\%). For subject4, the overall accuracy with both sensors are not high, although it can be observed that Action $A$ can be recognized by $60 \%$ (gyroscope), Action B by $80 \%$, and Action $C$ by $50 \%$ (accelerometer). The low overall accuracy can be attributed to the fact that subject 4 was at an early stage of rehabilitation and the impaired arm being tested was not the naturally dominant arm, thereby resulting in poor repeatability.

The performance of the proposed clustering-MDC methodology was further compared against two well-known supervised learning algorithms: linear discriminant analysis (LDA) and support vector machines (SVMs). LDA was chosen in view of its low computational complexity and SVM known for producing high classification accuracy [22]. The average overall accuracy using LDA for four healthy subjects was $45 \%$ using accelerometer data and 53\% using gyroscope data. Correspondingly, for four stroke survivors, the average accuracy was $49 \%$ and $46 \%$ using accelerometer and gyroscope, respectively. Similarly, using SVM, for the healthy subjects, the average accuracy was $54 \%$ and $68 \%$ using accelerometer and gyroscope, respectively, whereas for stroke survivors, the results were 55\% and 50\% using accelerometer and gyroscope data. Across all test cases, none of the subjects had all three movements classified with a sensitivity higher than $60 \%$ using either of the learning algorithms, thereby proving the effectiveness of our proposed methodology [8].

\section{B. Synthesis and Layout}

The design was synthesized using STMicroelectronics 130$\mathrm{nm}$ technology library with a supply voltage of $1.08 \mathrm{~V}$ and frequency of $50 \mathrm{~Hz}$, where the synthesized design occupied an area of $242 \mathrm{~K}$ (two-input NAND gate equivalent) and the dynamic power consumed was $5.3 \mathrm{nW}$. The design was also synthesized and functionally verified at a higher clock frequency of $20 \mathrm{MHz}$. The implementation of the feature extraction engine takes a maximum of $3 n$ clock cycles [17] (where $n$ is the number of input data samples), if it has to compute the all the ten features. The MDC design takes $(9 n+31)$ clock cycles in the worst case, considering it has to compute all the 30 features from the testing data set and compute the Euclidean distance to the three cluster centroids. To estimate the total chip area, a layout of the synthesized design was performed using the Cadence Encounter tool as

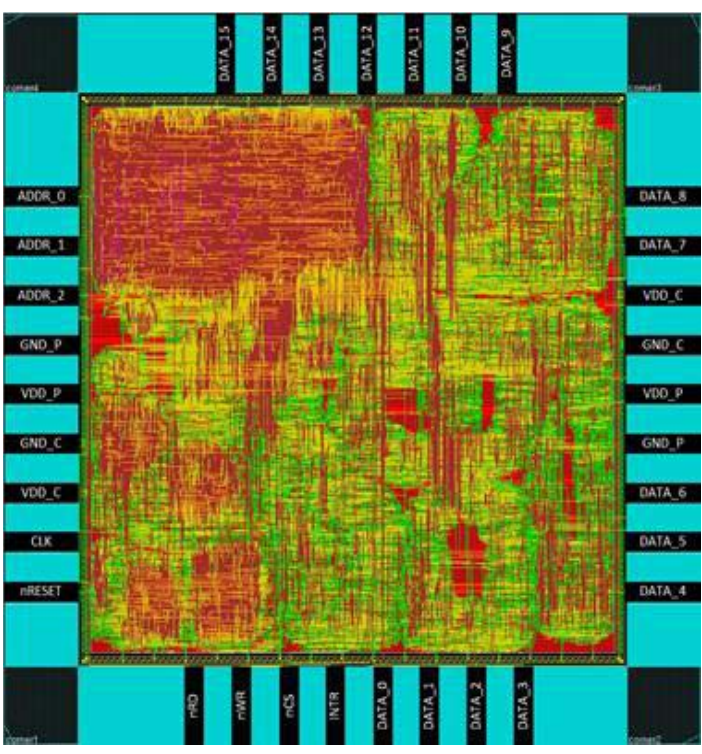

Fig. 8. Core chip layout with all pin assignments.

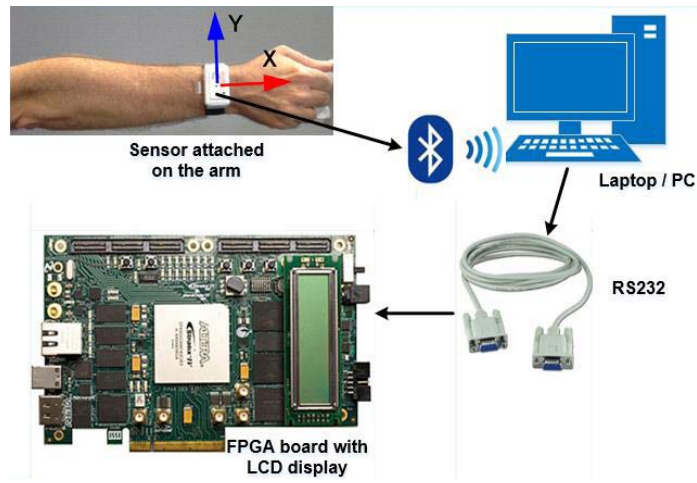

Fig. 9. FPGA-based demonstrator for real-time arm movement classification with movement data collected from the sensor attached to the arm.

shown in Fig. 8. The total area of the chip was estimated as $2.221 \mathrm{~mm} \times 2.215 \mathrm{~mm}$, having 25 signal pads and eight power/ground pads. The 16-bit input/output port is used for: 1) $i / p$-three sensor data streams (AccX, AccY, AccZ or GyroX, GyroY, GyroZ) sequentially; 2) $i / p$-three centroids; 3) $i / p$ - 30-bit feature code split into lower 16 bits followed by the higher 14 bits (padded with two zeroes); and 4) $o / p-2$ bits (padded with 14 zeroes) signifying the predicted cluster label.

\section{System Demonstrator Using FPGA}

The arm movement recognition framework (see Section II) has been demonstrated as a prototype system using an Altera DE4 FPGA in conjunction with a wrist-worn inertial sensor. The hardware setup for real-time implementation is shown in Fig. 9 where the data from the sensors (triaxial accelerometers) are transmitted to a host computer (i.e., PC) through Bluetooth. The raw data are converted to physical values and transmitted to the FPGA through RS232. The synthesized MDC HDL was integrated with RTL implementation of the RS232 receiver to complete the hardware functionality on the FPGA. 
The framework was validated with healthy subject2 performing one trial of 'making cup of tea,' where 18 out of the 20 movements were successfully detected. The three centroids are stored as binary data in the memory using the megafunction in Quartus, which allows the creation of a module that takes as input memory initialization files [23] and stores the data into the ROM of the FPGA. The feature code is sent using the synthesizable 'readmemb' function. The FPGA operates at a much higher frequency $(780 \mathrm{KHz}$ obtained through a clockdivider module) compared with the streaming sensor operating at $50 \mathrm{~Hz}$. The sensor data were communicated to the host PC through Bluetooth using the application ShimmerConnect [15]. The serial port control [24] was achieved through the .NET 4.5 framework and an application software (written in $\mathrm{C \#}$ ). The baud rate for transmitting the data from the PC to the FPGA was set to $4800 \mathrm{bits} / \mathrm{s}$, where each set of data was of 64 bits (16 bits for each $X, Y$, and $Z$ axes and header code). The start of transmission was indicated by the header code that helps the receiver determine the correct axes' value. A baud tick generator on the FPGA is used for interface synchronization that produces a pulse based on a counter logic. The classified arm movements are displayed on a seven-segment display in real time (Action $A-1, B-2$, and $C-3$ ). The synthesized design uses 40753 logic units and 13184 bits of memory (for storing the centroid and input triaxial data). The prototype takes 515 clock cycles $(\approx 0.6 \mathrm{~ms})$ to produce the desired output since it computes two features for healthy subject2 (std takes $2 n=512$ clock cycles) from the test data. For this demonstrator, we have used data from only one sensor type (i.e., accelerometers). However, this can be easily extended to incorporate the gyroscopes and the whole operation of 'feature extraction-MDC' can be independently performed on both sets of data to obtain the desired arm movement classification, and these results can be analyzed in line with the conclusions drawn in [8] and also in Section IV-A where considering more than one sensor type has been advocated to ascertain impaired arm usage and rehabilitation progress.

\section{RELATED WORK}

Real-time AR in body sensor networks is a challenging task, and energy efficiency has received particular attention in recent years from the pervasive computing research community for ways to extend the battery life of sensors aimed at long-term monitoring. With the advent of context-aware processing, energy-efficient processing on sensor nodes and mobile devices has taken precedence. A few recent papers [25]-[27] have discussed the need for reducing energy incurred on communication, with [27] showing the importance of on-node sensor processing over an off-node scheme saving up to $40 \%$ of energy trading off accuracy. Some of the recent online AR methods have looked into this aspect by processing on the sensors (e.g., low-power MSP430 microcontroller) or mobile phones (e.g., android) [27]. Another recent work [28] takes a hierarchical approach whereby they recognize hand gestures on the accelerometer sensor node using a Java-based simulator, but use this information to classify high-level activities on a mobile device by transmitting data through a wireless link. Apart from reducing communication (through on-node data processing and advocating light-weight algorithms), the focus has been on issues such as deactivation of power hungry sensors [29] (e.g., gyroscopes) and adaptive sampling rate [30]. Hence, to the best of our knowledge, this is the first work that has focused on an optimized low-complexity algorithmto-architecture mapping aimed toward a hardware/acceleratorbased design to be used within resource constrained senor nodes. Further energy saving design optimizations such as dynamic power management (for, e.g., shutting down feature extraction engine during MDC) and clock gating techniques can be incorporated to enhance the proposed low-complexity implementation.

\section{DISCUSSION}

In this paper, we have presented the architecture and implementation of a low-complexity framework for arm movement classification in an out-of-laboratory environment using bodyworn inertial sensors. A completely personalized approach has been presented, and the results obtained have been encouraging and show that these particular arm movements can be reliably detected with stroke survivors exhibiting moderate levels of involuntary tremor in their movements. The framework was further demonstrated as a proof-of-concept real-time arm movement recognition system.

One of the key features in such a system is the need for adaptability that caters to the change in movement patterns over time pertaining to each patient, thereby reflecting the improvement in their motor functionality as a result of the undergoing rehabilitation protocol. The demonstrated methodology can detect the change in movement patterns over a longitudinal scale by two means: 1) with decreasing movement recognition rates over time-due to the differing patterns of the daily life movements with respect to the precomputed cluster centroids in the selected feature space and 2) clinical intervention-clinicians observe a considerable change in the movements performed by the patients in comparison with their previous assessment (the time of obtaining the training data for the clusters). In such circumstances, the patient's training data would be collected periodically and the cluster centroids and the associated features (new selected feature set) can be recomputed to reflect the changing movement patterns. The new cluster centroids and feature set will be subject specific due to the intersubject variability inherent within movement profiles, variation in the rehabilitation profile, and the associated functional ability of each individual subject. This information could be further used by the MDC to recognize movements performed in daily life. Hence, we plan to carry out a longitudinal study in the near future to demonstrate the methodology for indicating rehabilitation progress.

In view of the designed architecture, there are a few fundamental factors that can be considered in future designs. First, the size of the register bank to store the incoming data samples from the sensors has been fixed at 256, representing $5 \mathrm{~s}$ of kinematic data (sensor streaming $50 \mathrm{~Hz}$ ). This time duration is suitable for the healthy subjects for the completion of the elementary arm movements (actions) chosen for the experimental protocol. For patients, depending on the level of dexterity, 
the time taken to perform the movements might be more especially when they are in their initial stage of rehabilitation. The next available window size in view of representing it in dyadic scale is 512 implying $10 \mathrm{~s}$ and would suit the requirements of patients needing more time to complete the actions. An alternate approach would be to reduce the sampling frequency in the range of 20-25 Hz, which has also been considered to be suitable in human AR [9], [10]. Second, here we consider the Euclidean distance over the Mahalanobis distance [8] for the MDC as a proof-of-concept implementation since the later increases the complexity involved in computing the covariance matrix. Third, a fundamental exploration in terms of error accumulation and propagation needs to be carried out, and accordingly, the datapath adjustment for ASIC implementation needs to be done in view of the target accuracy.

This design can be implemented as an ASIC chip and embedded on a sensor platform along with other processing components like A/D converter, filtering circuit, memory, and power source, to be used for real-time AR. An ASIC would provide leverage in terms of area and power compared with state-of-the-art microcontroller/mobile-platformbased designs, aiding the development of a point-of-care monitoring system. This methodology could be extended for lower limb monitoring and used with patients suffering from other neurodegenerative disorders exhibiting movement profiles that are less fluidic in nature. Real-time detection of arm movements can be useful in a wide array of applications in the field of sports, human computer interaction, or other treatments of arm dexterity. Therefore, the developed system can be used to track movements of required body segments in these respective fields outside a controlled environment.

\section{REFERENCES}

[1] F. E. Martínez-Pérez, J. Á. González-Fraga, J. C. Cuevas-Tello, and M. D. Rodríguez, "Activity inference for ambient intelligence through handling artifacts in a healthcare environment," Sensors, vol. 12, no. 1, pp. 1072-1099, 2012.

[2] B. Najafi, K. Aminian, A. Paraschiv-Ionescu, F. Loew, C. J. Bula, and P. Robert, "Ambulatory system for human motion analysis using a kinematic sensor: Monitoring of daily physical activity in the elderly," IEEE Trans. Biomed. Eng., vol. 50, no. 6, pp. 711-723, Jun. 2003.

[3] C. Zhu and W. Sheng, "Motion- and location-based online human daily activity recognition," Pervasive Mobile Comput., vol. 7, no. 2, pp. 256-269, 2011.

[4] L. Chen, J. Hoey, C. D. Nugent, D. J. Cook, and Z. Yu, "Sensor-based activity recognition," IEEE Trans. Syst., Man, Cybern. C, Appl. Rev., vol. 42, no. 6, pp. 790-808, Nov. 2012.

[5] J. Parkka, M. Ermes, P. Korpipaa, J. Mantyjarvi, J. Peltola, and I. Korhonen, "Activity classification using realistic data from wearable sensors," IEEE Trans. Inf. Technol. Biomed., vol. 10, no. 1, pp. 119-128, Jan. 2006.

[6] K. Altun, B. Barshan, and O. Tunçel, "Comparative study on classifying human activities with miniature inertial and magnetic sensors," Pattern Recognit., vol. 43, no. 10, pp. 3605-3620, Oct. 2010.

[7] K. Maharatna, E. B. Mazomenos, J. Morgan, and S. Bonfiglio, "Towards the development of next-generation remote healthcare system: Some practical considerations," in Proc. IEEE Int. Symp. Circuits Syst. (ISCAS), May 2012, pp. 1-4.

[8] D. Biswas et al., "Recognizing upper limb movements with wrist worn inertial sensors using k-means clustering classification," Human Movement Sci., vol. 40, pp. 59-76, Apr. 2015.
[9] M. Ermers, J. Pärkkä, J. Mäntyjärvi, and I. Korhonen, "Detection of daily activities and sports with wearable sensors in controlled and uncontrolled conditions," IEEE Trans. Inf. Technol. Biomed., vol. 12, no. 1, pp. 20-26, Jan. 2008.

[10] S. Chernbumroong, S. Cang, A. Atkins, and H. Yu, "Elderly activities recognition and classification for applications in assisted living," Expert Syst. Appl., vol. 40, no. 5, pp. 1662-1674, Apr. 2013.

[11] C. S. Lányi, V. Szücs, T. Dömok, and E. László, "Developing serious game for victims of stroke," in Proc. 9th Int. Conf. Disab., Virtual Reality Assoc. Technol., 2012, pp. 503-506.

[12] S. Ma, M. Varley, L.-K. Shark, and J. Richards, "Overcoming the information overload problem in a multiform feedback-based virtual reality system for hand motion rehabilitation: Healthy subject case study," Virtual Reality, vol. 16, no. 4, pp. 325-334, 2012.

[13] D. Biswas et al., "On the data analysis for classification of elementary upper limb movements," Biomed. Eng. Lett., vol. 4, no. 4, pp. 403-413, 2014.

[14] L. Bao and S. S. Intille, "Activity recognition from user-annotated acceleration data," in Pervasive Computing. Berlin, Germany: Springer, 2004, pp. 1-17.

[15] A. Burns et al., "SHIMMER-A wireless sensor platform for noninvasive biomedical research," IEEE Sensors J., vol. 10, no. 9, pp. $1527-1534$, Sep. 2010 .

[16] C. Kendell and E. D. Lemaire, "Effect of mobility devices on orientation sensors that contain magnetometers," J. Rehabil. Res., vol. 46, no. 7, pp. 957-962, 2009.

[17] D. Biswas and K. Maharatna, "A CORDIC-based low-power statistical feature computation engine for WSN applications," Circuits, Syst., Signal Process., vol. 34, no. 12, pp. 4011-4028, 2015.

[18] P. K. Meher, J. Valls, T.-B. Juang, K. Sridharan, and K. Maharatna, "50 years of CORDIC: Algorithms, architectures, and applications," IEEE Trans. Circuits Syst. I, Reg. Papers, vol. 56, no. 9, pp. 1893-1907, Sep. 2009.

[19] S. Patel et al., "A novel approach to monitor rehabilitation outcomes in stroke survivors using wearable technology," Proc. IEEE, vol. 98, no. 3, pp. 450-461, Mar. 2010.

[20] K. N. Vijeyakumar, V. Sumathy, P. Vasakipriya, and A. D. Babu, "FPGA implementation of low power high speed square root circuits," in Proc. IEEE Int. Conf. Comput. Intell. Comput. Res. (ICCIC), Dec. 2012, pp. $1-5$.

[21] L. Vachhani, K. Sridharan, and P. K. Meher, "Efficient CORDIC algorithms and architectures for low area and high throughput implementation," IEEE Trans. Circuits Syst. II, Express Briefs, vol. 56, no. 1, pp. 61-65, Jan. 2009.

[22] T. Chen, E. B. Mazomenos, K. Maharatna, S. Dasmahapatra, and M. Niranjan, "Design of a low-power on-body ECG classifier for remote cardiovascular monitoring systems," IEEE Trans. Emerg. Sel. Topics Circuits Syst., vol. 3, no. 1, pp. 75-85, Mar. 2013.

[23] Memory Initialization File, accessed on Jun. 12, 2015. [Online]. Available: http://quartushelp.altera.com/15.0/mergedProjects/reference/ glossary/def_mif.htm

[24] SerialPort Class, accessed on Jun. 2015. [Online]. Available: http:// $\mathrm{msdn} . \mathrm{microsoft} . \mathrm{com} / \mathrm{en}-\mathrm{us} / \mathrm{library} / \mathrm{system}$.io.ports.serialport $\% 28 \mathrm{v}=\mathrm{vs}$. 110\%29.aspx

[25] O. D. Lara and M. A. Labrador, "A survey on human activity recognition using wearable sensors," IEEE Commun. Surveys Tut., vol. 15, no. 3, pp. 1192-1209, 3rd Quart., 2013.

[26] T. Rault, A. Bouabdallah, Y. Challal, and F. Marin, "A survey of energy-efficient context recognition systems using wearable sensors for healthcare applications," Pervasive Mobile Comput., Aug. 2006. [Online]. Available: http://dx.doi.org/10.1016/j.pmcj.2016.08.003

[27] N. Wang, G. V. Merrett, R. G. Maunder, and A. Rogers, "Energy and accuracy trade-offs in accelerometry-based activity recognition," in Proc. 22nd Int. Conf. Comput. Commun. Netw., Nassau, Bahamas, Jul. 2013, pp. $1-6$.

[28] L. Wang, T. Gu, X. Tao, and J. Lu, "A hierarchical approach to realtime activity recognition in body sensor networks," Pervasive Mobile Comput., vol. 8, no. 1, pp. 115-130, Feb. 2012.

[29] K. Lorincz et al., "Mercury: A wearable sensor network platform for high-fidelity motion analysis," in Proc. 7th ACM Conf. Embedded Netw. Sensor Syst., Berkeley, CA, USA, 2009, pp. 183-196.

[30] X. Qi, M. Keally, G. Zhou, Y. Li, and Z. Ren, "AdaSense: Adapting sampling rates for activity recognition in body sensor networks," in Proc. 19th IEEE Real-Time Embedded Technol. Appl. Symp., Philadelphia, PA, USA, Apr. 2013, pp. 163-172. 


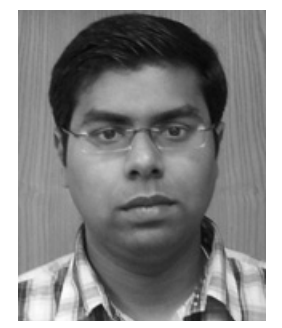

Dwaipayan Biswas received the M.Sc. and Ph.D. degrees from the University of Southampton, Southampton, U.K., in 2011 and 2015, respectively.

He is currently a Research Fellow with the University of Southampton, focusing on biomedical signal processing and embedded systems.

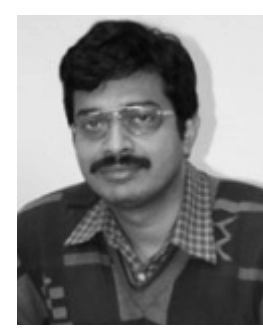

Koushik Maharatna (M'01) received the Ph.D. degree from Jadavpur University, Kolkata, India, in 2002. From 2000 to 2003, he was a Research Scientist with Innovations for High Performance, Frankfurt (Oder), Germany. He joined as a Lecturer Department of Electrical and Electronic Engineering, University of Bristol, in 2003. He has been with the School of Electronics and Computer Science, University of Southampton, Southampton, U.K., since 2006, where he is currently a Professor. His research interest includes biomedical signal processing, next generation healthcare systems development and VLSI design for signal processing systems.

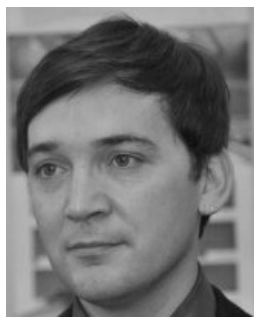

Goran Panic received the Ph.D. degree from the Brandenburg University of Technology Cottbus-Senftenberg, Senftenberg, Germany, in 2014.

$\mathrm{He}$ is currently a Scientist with the Systems Department of Innovations for High Performance, Frankfurt (Oder), Germany.

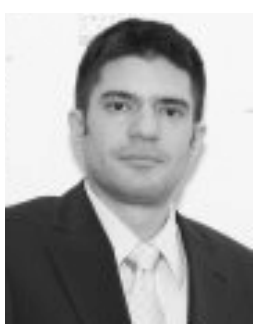

Evangelos B. Mazomenos received the Ph.D degree from the University of Southampton, Southampton, U.K., in 2012.

$\mathrm{He}$ is currently a Research Associate with the Centre for Medical Image Computing, University College London, London, U.K.

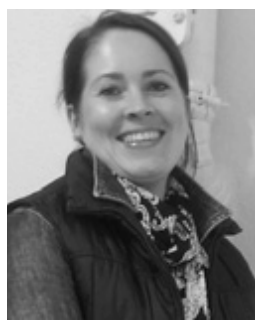

Josy Achner has been an Occupational Therapist and the Head of Occupational Therapy with the Neurologic Department, Brandenburg Klinik, Berlin, Germany, since 2002.

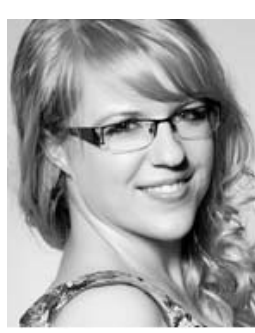

Jasmin Klemke has been an Occupational Therapist with the Neurologic Department, Brandenburg Klinik, Berlin, Germany, since 2006.

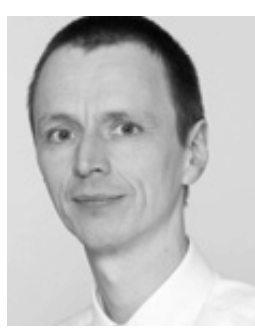

Michael Jöbges received the M.D. degree in neurophysiological from Medizinische Hochschule, Hannover, Germany in 1996.

$\mathrm{He}$ is currently a Consultant Neurologist and the Head of the Neurologic Department, Brandenburg Klinik, Berlin, Germany.

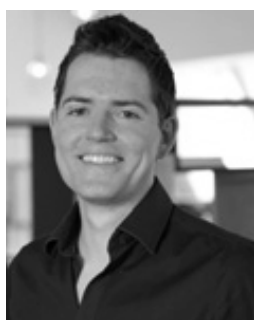

Steffen Ortmann received the Diploma in computer science and the Ph.D. degree with scholarship from the Technical University of Cottbus, Cottbus, Germany, in 2007 and 2010, respectively.

Since 2005, he has been with the Sensor Network Research Group, Innovations for High Performance, Frankfurt (Oder), Germany. 